\section{Promotionspreis der Deutschen Lungenstiftung „Untersuchungen zur Produktion von Stickstoffmonoxid in den menschlichen Atemwegen“}

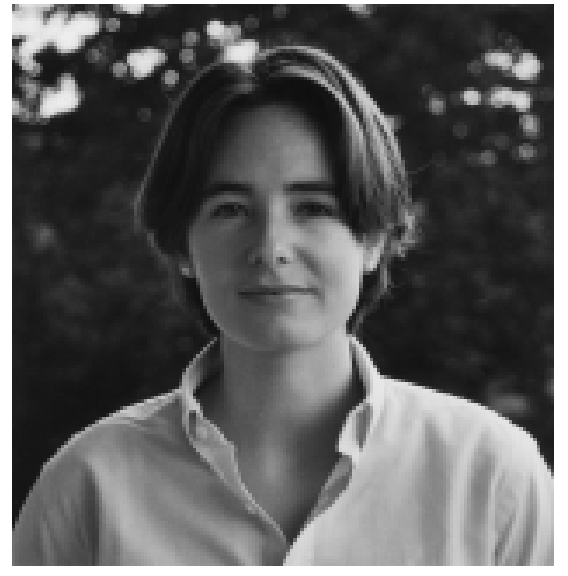

Anne-Marie Kirsten

\section{Einleitung}

Biologisch entspricht NO dem von Furchgott und Zawadzki (1980) beschriebenen „endothelium derived relaxing factor“ (EDRF). Im Jahre 1999 erhielten beide Autoren für diese Entdeckung den Nobelpreis. Erst 1987 erkannten zwei voneinander unabhängige Forschergruppen, dass es sich bei dem so genannten EDRF um Stickstoffmonoxid handelt. Seitdem konnte nachgewiesen werden, dass endogenes Stickstoffmonoxid eine Schlüsselrolle bei einer Vielzahl von Funktionen besitzt, u.a. der Regulation des Tonus der glatten Bronchialmuskulatur, bei Entzündungsreaktionen und der Mukussekretion.

Endogen produziertes NO ist ein Metabolit der halbessenziellen Aminosäure L-Arginin und wird mittels des Enzyms NOSynthase (NOS) produziert. Von diesem Enzym gibt es drei verschiedene Isoformen:

- Typ I (nNOS) in Neuronen

- Typ II (iNOS) in verschiedenen Entzündungszellen

- Typ III (ecNOS) in Endothelzellen

Alle drei Isoformen sind im menschlichen Respirationstrakt nachgewiesen. Selektive Inhibitoren der NOS sind bekannt. So ist zum Beispiel Aminoguanidin ein selektiver Inhibitor der induzierbaren Form der NOS (Typ II), $\mathrm{N}^{\mathrm{G}}$-nitro-L-arginine methyl ester (L-NAME) ist ein nicht-selektiver Inhibitor der NOS.

NO wird von verschiedenen Zellen des Respirationstraktes gebildet, wie Epithelzellen, Endothelzellen und Makrophagen. Dabei ist das Ausmaß der NO-Produktion durchaus verschieden. NO ist bei Patienten mit entzündlichen Erkrankungen in erhöhten Konzentrationen messbar z.B. beim Asthma bronchiale oder bei Atemwegsinfektionen. Es wird in der Nase, den Nasennebenhöhlen und der Lunge gebildet. Die in der Literatur vorgestellten Verfahren zur Messung von NO im Atemtrakt unterscheiden sich stark voneinander. Es wurde gezeigt, dass in der Nase speziell, vor allem im Nasopharynx und den Nasennebenhöhlen, verglichen mit den Bronchien, sehr hohe NO-Konzentrationen auftreten. Somit stellt eine Kontamination durch nasale Beimengungen bei der Analyse

Pneumologie 2000; 54: $561-563$

(c) Georg Thieme Verlag Stuttgart · New York ISSN 0934-8387 bronchialer NO-Konzentrationen ein besonderes Problem dar. Deshalb ist es wichtig zu wissen, ob eventuelle Unterschiede der nasalen NO-Produktion zwischen Gesunden und Asthmatikern bestehen, die auf eine simultane oder ggf. unterschiedliche Aktivierung der Zellen des oberen und des unteren Atemtraktes zurückzuführen sind. Ungeklärt war bis zur gegenwärtigen Arbeit weiterhin, inwieweit die schwer zu vermeidbaren nasalen Kontaminationen Unterschiede der bronchialen Produktion vortäuschen oder verwischen können. Ferner war nicht klar, in welcher Beziehung das ausgeatmete NO zu den in der bronchialen Schleimhaut verfügbaren Mengen von NO steht, insbesondere, ob bei langen Atemanhaltezeiten Sättigungsphänomene auftreten.

Es gibt Hinweise dafür, dass die Inhalation von Endotoxinen mit akuten und chronischen Atemwegsentzündungen einhergeht. Besonders Landwirte sind großen Mengen von Stäuben und Bakterien, welche Endotoxine enthalten, ausgesetzt. Diese Exposition scheint mit der Prävalenz und Inzidenz von Atemwegserkrankungen zu korrelieren. Die experimentelle Exposition gegenüber Endotoxinen verursacht eine Vermehrung der neutrophilen Granulozyten in der Lavage. Die Technik der Nasenlavage erlaubt es, Entzündungsmechanismen wenig invasiv in den oberen Atemwegen zu erfassen. So wurde gezeigt, dass die nasale Provokation mit endotoxinhaltigen Schweinestallstäuben eine sensitive Methode zum Nachweis der Antwort auf Endotoxine ist. Dieses Provokationsmodell kann möglicherweise durch Analyse der NOProduktion nach dem Provokationsreiz neue Erkenntnisse über Ausmaß und Dauer der Entzündung vermitteln.

\section{Fragestellungen}

In der Literatur der letzten Jahre wurde über erhöhte NOKonzentrationen im Exhalat bei Patienten mit Asthma berichtet. Unklar blieben neben messtechnischen Problemen u.a. der Anteil nasaler und bronchialer Produktionsraten, die Schleimhautkonzentration von NO im Vergleich zur Ausatemluft, sowie die NO-Antwort nach kurzzeitigen entzündlichen Reizen auf die Atemwege. Die Untersuchungen sollten deshalb der Beantwortung folgender Fragen dienen: 
- Gibt es Unterschiede in der Rate der bronchialen und nasalen Produktion von NO zwischen Gesunden und Asthmatikern?

- Wie verhält sich die NO-Konzentration in Abhängigkeit vom exspiratorischen Fluss?

- Kann man die NO-Antwort durch spezifische Entzündungsreize modulieren? Spiegelt sich die akute entzündliche Wirkung der nasalen Provokation mit Endotoxin auch in einer direkt messbaren Änderung der NO-Konzentration in der Nase wider?

- In welcher Weise beeinflussen inhalative Kortikosteroide die gemessenen nasalen NO-Konzentrationen?

\section{Versuchsaufbau (Abb.1)}

Die folgende Abbildung verdeutlicht exemplarisch die Methoden der verschiedenen Versuche. Es zeigt die Messung der gemischt nasal-bronchialen NO-Konzentrationen zur Bestimmung der Produktionsraten von Stickstoffmonoxid. In einem analogen Verfahren wurden während aktiven Absaugens der Luft aus dem Nasenraum die Produktionsraten von bronchial produziertem NO gemessen. Die Differenz erlaubte die Bestimmung der nasalen NO-Produktion unter vergleichbaren Versuchsbedingungen. Ferner wurde die Flussabhängigkeit des exhalierten NO untersucht. Durch die Einbringung von Widerständen in den Exspirationsschenkel kommt es zum Anlegen des Velums an die Rachenwand und so zum Abschluss der oberen Atemwege. Zusätzlich wurde durch dieses Verfahren eine Konstanthaltung des exspiratorischen Flusses erreicht.

\section{Ergebnisse}

Es zeigte sich, dass bei allen untersuchten Kollektiven die nasalen NO-Konzentrationen um ca. 2 Zehnerpotenzen höher lagen als die bronchial gemessenen NO-Konzentrationen. Nach nasaler Stimulation mit endotoxinhaltigen Stäuben zeigt sich über ein Zeitintervall von 3 Stunden ein geringer, aber signifikanter Anstieg der NO-Konzentration nach 30 Minuten.

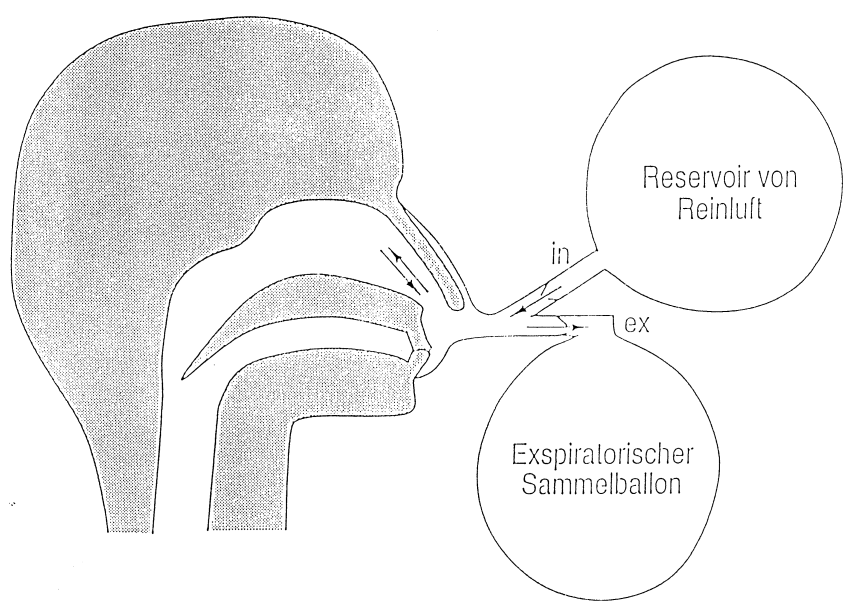

Abb. 1
Abb. 2 zeigt die Ergebnisse von Messungen der bronchialen NO-Konzentration bei verschiedenen, definierten, exspiratorischen Flussraten. Aufgetragen ist die NO-Konzentration gegen die Flussrate gemessen bei Gesunden (offene Quadrate), leichtgradigen Asthmatikern ohne Kortikosteroidtherapie (geschlossene Kreise) und mittel- bis schwergradigen Asthmatikern mit Kortikosteroidtherapie (offene Kreise). Die NOKonzentrationen der Asthmatiker, welche mit inhalativen Kortikosteroiden behandelt wurden, glichen fast denen der Gesunden, Probanden mit Asthma bronchiale ohne Kortikosteroidtherapie, zeigten jedoch um ein Vielfaches höhere NOKonzentrationen.

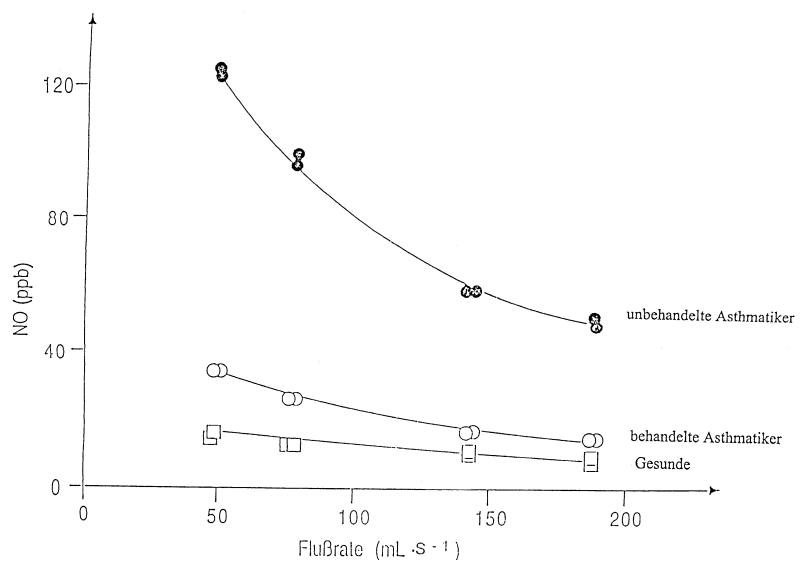

Abb. 2

\section{Einfluss der Kortikosteroide auf die NO-Produktion in den Atemwegen}

Es ist bekannt, dass die Inhalation von Kortikosteroiden bei Asthmatikern zu einer Reduktion der ausgeatmeten NOKonzentrationen führt. Kortikosteroide inhibieren die Expression der iNOS. Asthmatiker, welche regelmäßig Kortikosteroide benutzen, weisen eine deutlich geringere NO-Produktion gegenüber Asthmatikern ohne Kortikosteroide auf. Die hier gezeigten Daten in den vorbeschriebenen Versuchen stützen diese Befunde.

Durch die Messung des exhalierten NO scheint es in der Zukunft möglich, Patienten mit entzündlichen Atemwegserkrankungen zu erfassen und darüber hinaus den Therapieeffekt von Kortikosteroiden sowie die Compliance gegenüber Kortikosteroiden beim Asthma bronchiale zu quantifizieren.

Der Vergleich zwischen Asthmatikern mit und ohne Kortikosteroid-Therapie zeigte neben dem Effekt der Glukokortikoide auf die NO-Synthese keinen anderen messbaren Einfluss von Glukokortikoiden auf die Kinetik der NO-Produktion.

\section{Zusammenfassung}

Stickstoffmonoxid scheint eine bedeutende Rolle bei der Regulation des Tonus von Blutgefäßen und der Entzündung in den Atemwegen zu spielen. Untersuchungen der Ausatemluft mit Bestimmung des NO-Anteils bei gesunden Probanden und Patienten mit Asthma bronchiale zeigten erhöhte Werte bei 
den Patienten. Daraus kann auf eine pathophysiologische Bedeutung des NO bei entzündlichen Atemwegserkrankungen geschlossen werden.

Mit einem speziellen Messaufbau und einfachen Bilanzgleichungen gelang es uns erstmals, bei Gesunden sowie bei Asthmatikern mit und ohne inhalative Kortikosteroidtherapie die nasale und bronchiale NO-Produktion als Funktion der Atemanhaltezeit zu bestimmen. Dabei lagen die NO-Konzentrationen der Asthmatiker, die keine inhalativen Kortikosteroide erhielten, bronchial um den Faktor 3,5 und nasal um den Faktor 1,2 höher als diejenigen der Gesunden; entsprechend waren die Produktionsraten von NO im Zeitverlauf erhöht.

Die hier vorgelegten Untersuchungen ergaben eine deutliche Flussabhängigkeit des exhalierten NO. Dabei dienten verschiedene exspiratorische Widerstände zur Erzielung unterschiedlicher exspiratorischer Flussraten. Die Flussabhängigkeit des NO drückt sich grafisch näherungsweise in einer Exponentialfunktion aus. Die ausgeatmete NO-Konzentration ist nicht umgekehrt proportional dem Fluss, wie es bei einer konstanten NO-Produktion zu erwarten wäre, sondern zeigt eine geringe Variation. Mathematisch ist diese Variation als Abweichung der erwarteten Hyperbelfunktion zwischen NOProduktion und Fluss zu beschreiben. Dies wird auf die Tatsache zurückgeführt, dass die Größe des treibenden Konzentrationsgradienten von NO zwischen Schleimhaut und Luftraum durch Änderung des Flusses und damit der Konzentration im Luftraum geändert wird. Dies stellte einen indirekten Nachweis der bronchialen NO-Produktion dar.

Die Antwort der nasalen NO-Produktion auf einen spezifischen Entzündungsreiz wurde durch nasale Stimulation mit endotoxinhaltigen Stallstäuben untersucht. Hier kam es zu einem kurzfristigen Anstieg der NO-Produktion schon nach einer Stunde, welcher zeitlich dem Neutrophilen-Einstrom und der Hochregulation auf transkriptionaler Ebene vorrangeht. Somit scheint die Bestimmung von NO in der Atemluft ebenfalls geeignet, neben chronischen auch akute zelluläre Aktivierungen im Respirationstrakt zu erfassen.

Durch Untersuchungen von Probanden mit Asthma, welche Kortikosteroide inhalierten und Asthmatikern, die keine Kortikosteroide benutzten, konnte ebenfalls nachgewiesen werden, dass eine Kortikosteroidbehandlung in der Lage ist, die NO-Produktion signifikant zu senken $[1,2]$.

\section{Literatur}

${ }^{1}$ Kirsten AM, Jörres RA, Kirsten D, Magnussen H. Effect of nasal challenge with endotoxin-containing swine confinement dust on nasal nitric oxide production. Europ J Med Res 1997; 2: 335 339

${ }^{2}$ Kirsten AM, Jörres RA, Kirsten D, Magnussen H. Vergleich der nasalen und bronchialen Produktion von Stickstoffmonoxid bei Gesunden und Patienten mit Asthma. Pneumologie 1997; 51: $359-364$
Dr. med. A.-M. Kirsten

III. Medizinische Klinik Schwerpunkt Pneumologie Johannes-Gutenberg-Universität Mainz Langenbeckstr. 1

55131 Mainz 